\title{
Functional genomic screening to enhance oncolytic virotherapy
}

\author{
D J Mahoney ${ }^{1,2,3}$ and D F Stojdl ${ }^{\star, 4,5,6}$ \\ ${ }^{1}$ Alberta Children's Hospital Research Institute, Calgary, AB, Canada T2N 4N1; ${ }^{2}$ Southern Alberta Cancer Research Institute, \\ Calgary, AB, Canada T2N 4N1; ${ }^{3}$ Department of Microbiology, Immunology and Infectious Disease, Faculty of Medicine, University \\ of Calgary, Calgary, AB, Canada T2N 4N1; ${ }^{4}$ Children's Hospital of Eastern Ontario Research Institute, 401 Smyth Road, Ottawa, \\ ON, Canada K1H 8L1; ${ }^{5}$ Department of Pediatrics, University of Ottawa, Ottawa, ON, Canada K1H $8 \mathrm{~L} 1$ and ${ }^{6}$ Department of \\ Biochemistry, Microbiology, and Immunology, University of Ottawa, Ottawa, ON, Canada K1H $8 L 1$
}

Functional genomic screening has emerged as a powerful approach for understanding complex biological phenomena. Of the available tools, genome-wide RNA interference (RNAi) technology is unquestionably the most incisive, as it directly probes gene function. Recent applications of RNAi screening have been impressive. Notable amongst these are its use in elucidated mechanism(s) for signal transduction, various aspects of cell biology, tumourigenesis and metastasis, resistance to cancer therapeutics, and the host's response to a pathogen. Herein we discuss how recent RNAi screening efforts have helped turn our attention to the targetability of non-oncogene support pathways for cancer treatment, with a particular focus on a recent study that identified a non-oncogene addiction to the ER stress response as a synergist target for oncolytic virus therapy (OVT). Moreover, we give our thoughts on the future of RNAi screening as a tool to enhance OVT and describe recent technical improvements that are poised to make genome-scale RNAi experiments more sensitive, less noisy, more applicable in vivo, and more easily validated in clinically relevant animal models.

\section{INTRODUCTION: THE COMING OF AGE OF RNAi SCREENING TECHNOLOGY}

The landmark discovery of RNA interference (RNAi) as a mechanism for gene regulation in eukaryotic cells was a defining scientific achievement of the past two decades. Equally impressive was the technologisation of RNAi that rapidly followed, which has revolutionised the research process for the biological sciences. Reverse genetic experiments using RNAi tools are extremely informative and usually incisive, as well as inexpensive, quick, and easy to perform. Advances in delivery vectors have enabled RNAi experiments in almost all cell types, both in vitro and increasingly in vivo. Importantly, the acquisition, annotation, and accessibility of the complete genome sequences for humans and commonly used model organisms has greatly expanded the utility of RNAi reagents. Not only has this genomic information vastly broadened the usability of RNAi technology in reductionist-type experiments, but also it has allowed for the adaptation of RNAi tools for largescale forward genetic screens.
Nearing a decade old and ever evolving, genome-wide RNAi technology has certainly come of age. The fruit borne from recent screens are plentiful as follows: novel mechanisms elucidated in signal transduction pathways, such as those involved in the DNA damage response (Cotta-Ramusino et al, 2011), integrin (Rantala et $a l, 2011$ ), and NF- $\kappa \mathrm{B}$ (Murphy et al, 2011) signalling; new insights into fundamental biological processes, such as antigen presentation (Paul et al, 2011), autophagy (Orvedahl et al, 2011), and pluripotency (Chia et al, 2010); identification of therapeutic targets for pathological processes, such as tumourigenesis (Kessler et al, 2012), tumour maintenance (Zuber et al, 2011b), and resistance to cancer treatment (Giamas et al, 2011); and the annotation of hundreds of biological contact points between host organisms and pathogenic viruses (reviewed in Friedel and Haas, 2011). As a particularly illustrative example, Kessler et al (2012) recently published tantalising data from a genome-wide RNAi screen probing human mammary epithelial cells for 'nononcogenic support pathways' for the $M y c$ oncogene (Kessler et al, 2012). Dysregulated in $\sim 25 \%$ of breast cancers, Myc is 
required for tumour maintenance and progression, and is associated with very poor prognosis; however, despite intensive efforts spanning nearly three decades there is no effective way to target Myc in human cancers. Taking a synthetic lethal RNAi screening approach, Kessler et al (2012) identified a SUMOylation-dependent transcriptional programme that is essential for Myc-induced tumourigenesis. In Myc-driven cancers, the authors showed that SUMO-activating enzyme 2 (SAE2) is required to maintain a supportive Myc transcriptional subprogramme in its activated state. Loss of SAE2 represses this programme and leads to mitotic collapse and cell death in tumours with hyperactivated Myc.

In the realm of cancer research, the work by Kessler et al (2012) highlights one of the great strengths of genome-scale RNAi screening: the ability to identify tumour vulnerabilities that are not oncogenic per se. Similar genome-scale synthetic lethal studies have been conducted with the frequently hyperactivated and difficult-totarget oncogene RAS, generating equally impressive results (Scholl et al, 2009). These studies illuminate a growing trend in cancer research: discovering and targeting non-oncogene addiction (NOA) in tumours (Luo et al, 2009). Since the discovery of the Src oncogene over four decades ago, small and large-scale sequencing efforts have been aimed towards identifying oncogenes in human cancers. As a collective, this pursuit has generated an unprecedented wealth of information. Hundreds of oncogenes have been catalogued and a staggering amount has been learned about the genesis and maintenance of human tumours. Unfortunately for cancer patients, this work has also taught us that oncogenes are not, generally speaking, useful therapeutic targets (Poulikakos et al, 2011). This great effort informed us that, with a few notable exceptions such as chronic myelogenous leukaemia, cancer is a disease of genetic and phenotypic heterogeneity, consisting of distinct cell populations that are maintained by a distinct set of oncogenic mutations (Snuderl et al, 2011). A drug that inhibits an individual oncogene, therefore, only targets a subset of the cancer's cells; in fact, as the cancer ecosystem is one where tumour cells are forever competing for resources, removing a sub-population of cancer cells can inadvertently facilitate the expansion of another, perhaps more resistant sub-population, a concept known as competitive release (Greaves and Maley, 2012). These research pursuits also taught us that cancer genomes are highly unstable and cellular signalling pathways are functionally degenerate. Consequently, the selective pressures applied by drugs targeting a single oncogene within a particular growth pathway invariably leads to adaptive tumour evolution and drug resistance (Zhang et al, 2010).

Fortunately, on the heels of these sobering realities a new paradigm has emerged that oncogenes themselves are dependent upon normal cellular signalling networks, and that cancer cells are dependent upon normal cellular stress responses (Solimini et al, 2007). These NOAs are not genetically mutated and, thus, would not be detected in an audit of a cancer's genome. They are, however, disproportionately critical to the viability of tumour as compared with normal cells. Emboldened by the prospect that these non-oncogenic support pathways may be common to multiple oncogenes, have fewer functional redundancies with which to permit a path for resistance, and offer multiple drug targets, many cancer scientists have begun to herald the pursuit of non-oncogene targets as the new path forward (Luo et al, 2009). By its very nature, genome-scale RNAi screening technology is tailor-made to discovering these critical supportive vulnerabilities in tumours.

RNAi SCREENING TO ENHANCE ONCOLYTIC VIRUS THERAPY FOR CANCER

High-throughput RNAi screening is also, as it turns out, tailormade to functionally annotate interactomes between host organisms and pathogenic viruses (reviewed in Friedel and Haas, 2011).
Somewhat analogous to the cancer story, an emerging movement in the infectious disease field has been to target host factors required for a productive virus infection. This therapeutic approach was borne out of realisation that virus factors, although conceptually attractive targets because they should, in theory, provide a wide therapeutic index, are challenging points of intervention because of the high mutability of their genomes. This is particularly true for RNA viruses, such as HIV, which requires a cocktail of drugs inhibiting multiple virus proteins for effective management. Targeting key host factors, on the other hand, may be a more tractable approach, as it should be more difficult for a virus to develop resistance. A major step forward for this idea occurred in 2007, when the FDA approved the chemokine (C-C motif) receptor 5 inhibitor, maraviroc, as the first-in-class drug targeting a host factor required for a pathogenic virus (HIV-1, R5 strain; Sax, 2007).

Given the extraordinary power of genome-scale RNAi screening for discovering cancer vulnerabilities and host factors for viral pathogens, and the attractiveness of targeting host factors to alter virus productivity, we have argued that a synthetic lethal RNAi screen in resistent cancer cells with a cancer-fighting OV should uncover unforeseen and therapeutically relevant biological nexuses between the host's tumour and the OV (Mahoney and Stojdl, 2010). Also undergoing a coming of age, OV therapy uses nonpathogenic or attenuated viruses that have natural or engineered tropism for tumour cells to treat cancer (reviewed in Auer and Bell, 2012). Three features make OVs particularly attractive cancer therapeutics. The first is that they seek out tumour cells based on downstream consequences of an oncogenic mutation, that is, altered molecular circuitry and/or NOA, such as attenuated type I interferon signalling, rather than targeting an oncogene per se (Stojdl et al, 2000). The second is that they lead to cancer cell killing through multiple mechanisms of action, such as directly lysing tumour cells (Stojdl et al, 2003), stimulating antitumour immunity (Diaz et al, 2007), and starving a cancer by infecting endothelial cells and inducing tumour vasculature collapse (Breitbach et al, 2007). The third is that they are programmable machines, that is, they can be genetically engineered in creative ways to enhance efficacy, such as arming them with the anti-tumour immune cytokine GM-CSF (Bristol et al, 2003). Collectively, these three features may help counter the evasive nature of malignancy that currently stymies existing cancer therapies. And with three OV platforms currently in phase II/b or III clinical evaluation for multiple cancer indications, it appears that oncolytic virus therapy (OVT) is poised to become an approved treatment option within the next 2-5 years (reviewed in Auer and Bell, 2012).

We recently published the first report of a genome-wide RNAi screen specifically designed to probe the interactome between cancer cells and an OV (Mahoney et al, 2011). Using the oncolytic rhabdovirus Maraba (Brun et al, 2010), we performed cell-based synthetic lethal analyses with three distinct cancer genomes, from which an intriguing concept unfolded; inducing a mild, transient stress by inhibiting a non-oncogene support pathway can lead to tumour-specific reprogramming that renders cancer cells susceptible to a partnered therapeutic agent. Specifically, we found that transiently blocking components of the ER stress response, a common NOA in many tumours, leads to a gradual accumulation of misfolded proteins in the ER. During the stress-management process that ensues, levels of the adaptor protein RAIDD (RIP associated Ich-1/CED homologous protein with death domain) rise, which opens the door for caspase-2-mediated apoptosis when subsequently challenged with the oncolytic Maraba virus (Figure 1). In terms of clinical implications, it is noteworthy that ER stress response inhibitors and Maraba virus are both currently in preclinical development. Moreover, we found that the clinically approved cytotoxic agent doxorubicin, known to rely on the 


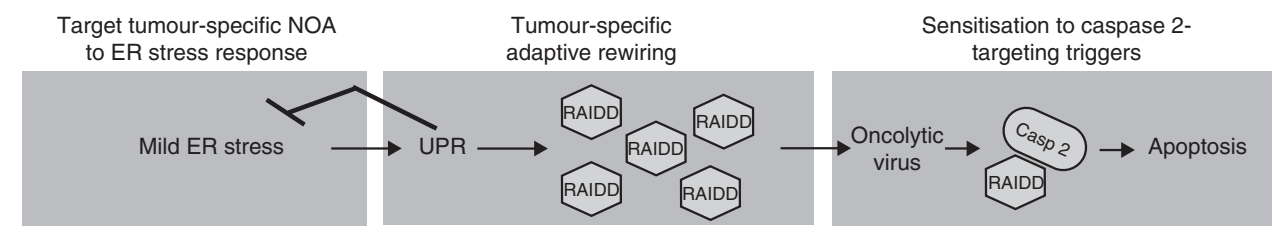

Figure 1. Targeting a tumour-specific NOA to the ER stress response induces cancer cell rewiring and sensitisation to caspase-2- and RAIDD-dependent viral oncolysis. A genome-scale RNAi screen uncovered that targeting a tumour-specific NOA to the ER stress response causes a mild ER stress that evokes a corrective UPR in tumour cells. As a consequence, levels of the adaptor protein RAIDD increase, which sensitises tumour cells to caspase-2-dependent apoptosis when challenged with a partnered therapeutic oncolytic virus. NOA, non-oncogene addiction; ER, endoplasmic reticulum; UPR, unfolded protein response.

caspase-2/RAIDD signalling axis to induce cancer cell death, is also synergised by pretreatment with ER stress response inhibitors.

One feature of this study particularly stands out; the therapeutic index that we uncovered was a product of targeting a NOA. This was not by design, but rather an inadvertent byproduct of the RNAi screening approach taken. On the basis of the rich hostpathogen interactome data that were being generated for other viruses in similarly executed RNAi screens, we had anticipated discovering novel host factors that repress virus productivity, which could thereafter be targeted for a therapeutic gain. Instead, we discovered that inhibiting a non-oncogene support pathway synergised with OV-mediated killing in an unusual way. In contrast to studies like those published by Kessler et al (2012), which identified NOA as a stand-alone cancer vulnerability, our work revealed that targeting the NOA to the ER stress response induces adaptive rewiring in cancer cells that renders them highly susceptible to OV-mediated killing. In essence, our study demonstrated the capacity to engineer a cancer vulnerability by targeting a NOA, which can subsequently be exploited by a partnered OV (Mahoney et al, 2011).

\section{RECENT ADVANCES IN GENOME-SCALE RNAi} SCREENING TECHNOLOGY

A number of technical innovations made over the past year are poised to improve genome-scale RNAi screening in three important ways. First, the development of next-generation inducible shRNA vectors and advances in mouse shRNA transgenic technology will make in vivo validation easier and more clinically relevant. Second, these new vector systems, coupled with recent advances in barcode deconvolution and bioinformatics, will enhance the utility of pooled shRNA screening in vivo. Third, the development of a massively parallel sensor assay for unbiased, high-throughput, functional evaluation of shRNA sequences is a prelude to the production of next-generation genome-wide shRNA resources that will be more potent and less noisy than the existing libraries.

NextGen shRNA vectors: implications for in vivo validation and pooled screening. Third-generation inducible shRNA vectors have recently been developed (Meerbrey et al, 2011; Zuber et al, 2011a). These expression vehicles, termed the TRMPV and pINDUCER series of vectors, offer one significant advantage over the first- and second-generation platforms, such as pSuperior.retro, Tet-pLKO-Puro, pLKO-puro-IPTG-1xLacO, pPRIME, pSLIK, and pTRIPZ (Hu and Luo, 2012). Both TRMPV and pINDUCER vectors are modular systems that have the following features: mir30-based shRNA sequences and a fluorescent reporter under the control of the tetracycline response element; the reverse tetracycline transactivator and either a fluorescent or luminescent reporter, or a selectable gene marker under the control of constitutively active promoters; the choice of a retrovirus- or lentivirus-based delivery system; and compatibility with the existing Hannon-Ellidge miR30-based shRNA libraries. Collectively, these features bestow the ability to (1) induce potent and reversible silencing of any human or mouse gene upon the onset of disease; (2) conduct experiments with mixed cell populations, drug-selected populations, or highly expressing cell clones; (3) silence genes in nearly any primary or immortalised cell of interest; and (4) simultaneously track tumour size and shRNA expression in vivo with dual fluorescent and luminescent reporters.

Regarding in vivo validation, these next-generation vector systems endow the capacity to rapidly evaluate a cancer gene target in nearly any xenograft or syngeneic transplantable tumour model. The major advantage over previous technologies is that following transduction with a single TRMPV or pINDUCER vector, cancer cells can be monitored in vivo post-transplantation and gene silencing can be induced and monitored after the onset of detectable disease. In this scenario, target validation more closely resembles the human clinical situation.

Of course, silencing a gene target in cancer established on a wild-type background has one obvious limitation: therapeutic index cannot be evaluated. Ideally, transduced cells would be transplanted into recipient mice that themselves have the gene target under the control of the same inducible promoter. This approach would more closely mimic the clinical situation where both the cancer and the host are dosed with a therapeutic agent. The International Knockout Mouse Consortium is diligently working towards generating a conditional knockout mouse anthology that spans the entire genome; however, until the inducible models come on line, new developments in transgenic RNAi mouse technology offer an exciting alternative. Using modified retroviral-based shRNA vectors, two studies have recently demonstrated the feasibility of engineering transgenic shRNA mice that possess inducible, reversible, trackable, and high-level expression of RNAi in vivo (McJunkin et al, 2011; Premsrirut et al, 2011). To be fair, these are not the first reports of inducible RNAi transgenic mice; however, their approach was developed to overcome the major limitation of the previous technology, that is, highly variable gene knockdown. By coupling a modified TMP shRNA vector to a Flp/FRT recombinase-mediated cassette exchange approach, the authors were able to reproducibly engineer single-copy, doxycycline-inducible RNAi transgenic embryonic stem cells with which to generate transgenic RNAi mice that had robust and reversible gene knockdown in vivo. With this advance, it is now theoretically possible to partner inducible gene knockdown in cancer cells with that in a recipient transgenic mouse, to fully recapitulate, genetically, the interactions between a drug, the cancer, and the host.

The ability of next-generation shRNA vectors to induce gene knockdown in transplantable cancer cells after the onset of disease in the recipient host is also an advantageous feature for in vivo pooled RNAi screening. Similar to its benefits for in vivo validation, silencing gene expression after disease onset is more likely to identify clinically useful cancer targets in an in vivo screen 
than an approach that uses stable gene silencing. To demonstrate proof-of-concept for using next-generation shRNA libraries for in vivo screening, Zuber et al (2011a) spiked a library of 820 TRMPV shRNA vectors with three potent shRNAs targeting the replication protein A (Rpa3), a gene whose knockdown causes cell cycle arrest in dividing cells (Zuber et al, 2011a). This pool was transduced into acute myeloid leukaemia (AML) cells, drug selected, and transplanted into recipient mice. After the onset of leukaemia, mice were left untreated or treated with doxycycline. After 10 days, AML cells were collected, sorted for Venus + dsRed + expression (i.e., cells that were transduced and expressing their shRNA), and identified by deep sequencing. When compared with AML cells extracted from control mice, those derived from doxycycline-treated animals showed a 4- to 10-fold depletion of TRMPV-Rpa3. Furthermore, all three Rpa3 shRNAs spiked into the library were among the top 25 hits from the screen. Taken together, these data highlight the added benefits of next-generation inducible shRNA vectors that, when coupled with several recent innovations in barcode deconvolution and bioinformatics (e.g., Sims et al, 2011), greatly improve the utility of pooled RNAi screens in vivo for the discovery of clinically relevant cancer gene targets.

RNAi design using a massively parallel sensor assay. As the requirements for efficient RNAi-mediated target suppression are not well known, shRNAs designed using the existing algorithms do not consistently knock down their target genes. To get around this knowledge gap, Fellmann et al (2011) recently developed an innovative, unbiased high-throughput 'sensor' assay to identify potent RNAi sequence on a large scale (Fellmann et al, 2011). This technology consists of a single vector (pSENSOR) reporter assay containing doxycycline-inducible miR30-based shRNA sequences and a constitutively active Venus reporter coupled to the cognate RNAi target sequence (i.e., the 'sensor'). The potency of a given shRNA sequence is evaluated by transducing cells with pSENSOR, dosing them with doxycycline, and monitoring the loss of Venus signal intensity (which will be degraded along with the sensor sequence). The major innovation here, when compared with other systems that similarly monitor RNAi potency, is that all the components are engineered into a single construct. This has two important implications as follows. (1) It allows the assay to be multiplexed. Similar to pooled RNAi screens, cells can be transduced at low multiplicity of infection to ensure a single integration per cell. Thousands of cells containing thousands of different shRNA sequences can then be combined, treated with doxycycline, and sorted by Venus intensity using FACS. Deep sequencing can subsequently identify the RNAi sequences that were particularly good at depleting the Venus signal strength. (2) Evaluating single-copy shRNA potency is highly relevant to the recent innovations in validation and pooled screening in vivo described above, which rely on potent single-copy gene silencing.

To test their system in high-throughput, Fellmann et al (2011) multiplexed nearly 20000 different shRNAs comprising every possible RNAi target sequence against 9 different genes. Following several rounds for FACS sorting of Venus-depleted cells, DNA sequencing confirmed the enrichment of 17 shRNA sequences that were previously known to potently induce gene silencing. Moreover, structure-function analyses of the 20000 sequences revealed several previously unknown properties that affect shRNA efficacy, which have subsequently been incorporated into a new algorithm for shRNA design.

\section{CONCLUSIONS: THE FUTURE OF RNAi SCREENING TO} ENHANCE OVT

With a single published report of a functional genomic screen to identify cancer-specific targets for improving OVT (Mahoney et al,

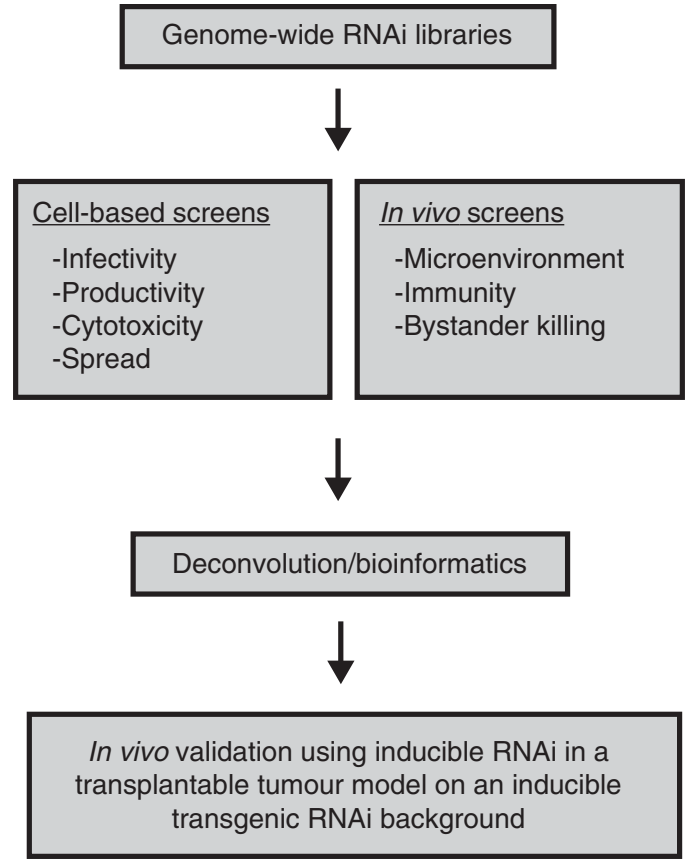

Figure 2. Pipeline for genome-wide RNAi screening to enhance OVT. Using genome-scale siRNA and shRNA libraries, both cell-based and in vivo screens can be used to catalogue the genes involved in many aspects of the tumour-OV-host interactomes. Following hit deconvolution and bioinformatic analyses, clinically relevant secondary in vivo validation can be performed using novel inducible RNAi reagents in xenografts or syngeneic transplantable tumour models on a matching inducible RNAi transgenic mouse. OVT, oncolytic virus therapy.

2011), and several important innovations in the shRNA field within the past 12 months, the future of RNAi screening to enhance OVT is extremely bright. We have previously outlined the points of intervention for OVT that can be studied using RNAi screening technology in cell-based experiments (Mahoney and Stojdl, 2010). Forthcoming next-generation libraries containing more potent and specific shRNAs will make these screens more sensitive and accurate at identifying these therapeutic opportunities. Moreover, next-generation shRNA vectors will facilitate the rapid and clinically relevant evaluation of the screening hits in animal models, as well as the identification of new targets in a broader array of clinically significant in vivo screens. As a case in point, these novel platform will enhance our capacity to identify, using in vivo RNAi screens, points of intervention that cannot be discovered in cell-based assays, such as modulators of the tumour microenvironment, tumour immunity, and bystander killing. Together, these innovations are poised to streamline the process of discovery and preclinical validation of targets that, when inhibited, synergise with OVT (Figure 2). As the clinical approval of the first-in-class OVT may be just around the corner, the time is ripe to leverage these genome-scale RNAi tools to enhance OVT.

\section{REFERENCES}

Auer R, Bell JC (2012) Oncolytic viruses: smart therapeutics for smart cancers. Future Oncol 8: 1-4.

Breitbach CJ, Paterson JM, Lemay CG, Falls TJ, McGuire A, Parato KA, Stojdl DF, Daneshmand M, Speth K, Kirn D, McCart JA, Atkins H, Bell JC (2007) Targeted inflammation during oncolytic virus therapy severely compromises tumor blood flow. Mol Ther J Am Soc Gene Ther 15: 1686-1693. 
Bristol JA, Zhu M, Ji H, Mina M, Xie Y, Clarke L, Forry-Schaudies S, Ennist DL (2003) In vitro and in vivo activities of an oncolytic adenoviral vector designed to express GM-CSF. Mol Ther J Am Soc Gene Ther 7: 755-764.

Brun J, McManus D, Lefebvre C, Hu K, Falls T, Atkins H, Bell JC, McCart JA, Mahoney D, Stojdl DF (2010) Identification of genetically modified Maraba virus as an oncolytic rhabdovirus. Mol Ther J Am Soc Gene Ther 18: 1440-1449.

Chia NY, Chan YS, Feng B, Lu X, Orlov YL, Moreau D, Kumar P, Yang L, Jiang J, Lau MS, Huss M, Soh BS, Kraus P, Li P, Lufkin T, Lim B, Clarke ND, Bard F, Ng HH (2010) A genome-wide RNAi screen reveals determinants of human embryonic stem cell identity. Nature 468 : 316-320.

Cotta-Ramusino C, McDonald III ER, Hurov K, Sowa ME, Harper JW, Elledge SJ (2011) A DNA damage response screen identifies RHINO, a 9-1-1 and TopBP1 interacting protein required for ATR signaling. Science 332: 1313-1317.

Diaz RM, Galivo F, Kottke T, Wongthida P, Qiao J, Thompson J, Valdes M, Barber G, Vile RG (2007) Oncolytic immunovirotherapy for melanoma using vesicular stomatitis virus. Cancer Res 67: 2840-2848.

Fellmann C, Zuber J, McJunkin K, Chang K, Malone CD, Dickins RA, Xu Q, Hengartner MO, Elledge SJ, Hannon GJ, Lowe SW (2011) Functional identification of optimized RNAi triggers using a massively parallel sensor assay. Mol Cell 41: 733-746.

Friedel CC, Haas J (2011) Virus-host interactomes and global models of virus-infected cells. Trends Microbiol 19: 501-508.

Giamas G, Filipovic A, Jacob J, Messier W, Zhang H, Yang D, Zhang W, Shifa BA, Photiou A, Tralau-Stewart C, Castellano L, Green AR, Coombes RC, Ellis IO, Ali S, Lenz HJ, Stebbing J (2011) Kinome screening for regulators of the estrogen receptor identifies LMTK3 as a new therapeutic target in breast cancer. Nat Med 17: 715-719.

Greaves M, Maley CC (2012) Clonal evolution in cancer. Nature 481: 306-313.

Hu G, Luo J (2012) A primer on using pooled shRNA libraries for functional genomic screens. Acta Biochim Biophys Sin (Shanghai) 44: 103-112.

Kessler JD, Kahle KT, Sun T, Meerbrey KL, Schlabach MR, Schmitt EM, Skinner SO, Xu Q, Li MZ, Hartman ZC, Rao M, Yu P, Dominguez-Vidana R, Liang AC, Solimini NL, Bernardi RJ, Yu B, Hsu T, Golding I, Luo J, Osborne CK, Creighton CJ, Hisenbeck SG, Schiff R, Shaw CA, Elledge SJ, Westbrook TF (2012) A SUMOylation-dependent transcriptional subprogram is required for Myc-driven tumorigenesis. Science 335: 348-353.

Luo J, Solimini NL, Elledge SJ (2009) Principles of cancer therapy: oncogene and non-oncogene addiction. Cell 136: 823-837.

Mahoney DJ, Lefebvre C, Allan K, Brun J, Sanaei CA, Baird S, Pearce N, Gronberg S, Wilson B, Prakesh M, Aman A, Isaac M, Mamai A, Uehling D, Al-Awar R, Falls T, Alain T, Stojdl DF (2011) Virus-tumor interactome screen reveals ER stress response can reprogram resistant cancers for oncolytic virus-triggered caspase- 2 cell death. Cancer Cell 20: 443-456.

Mahoney DJ, Stojdl DF (2010) A call to arms: using RNAi screening to improve oncolytic viral therapy. Cytokine Growth Factor Rev 21: 161-167.

McJunkin K, Mazurek A, Premsrirut PK, Zuber J, Dow LE, Simon J, Stillman B, Lowe SW (2011) Reversible suppression of an essential gene in adult mice using transgenic RNA interference. Proc Natl Acad Sci USA 108: 7113-7118.

Meerbrey KL, Hu G, Kessler JD, Roarty K, Li MZ, Fang JE, Herschkowitz JI, Burrows AE, Ciccia A, Sun T, Schmitt EM, Bernardi RJ, Fu X, Bland CS, Cooper TA, Schiff R, Rosen JM, Westbrook TF, Elledge SJ (2011) The pINDUCER lentiviral toolkit for inducible RNA interference in vitro and in vivo. Proc Natl Acad Sci USA 108: 3665-3670.

Murphy SH, Suzuki K, Downes M, Welch GL, De Jesus P, Miraglia LJ, Orth AP, Chanda SK, Evans RM, Verma IM (2011) Tumor suppressor protein (p)53, is a regulator of NF-kappaB repression by the glucocorticoid receptor. Proc Natl Acad Sci USA 108: 17117-17122.

Orvedahl A, Sumpter Jr R, Xiao G, Ng A, Zou Z, Tang Y, Narimatsu M, Gilpin C, Sun Q, Roth M, Forst CV, Wrana JL, Zhang YE, Luby-Phelps K, Xavier RJ, Xie Y, Levine B (2011) Image-based genome-wide siRNA screen identifies selective autophagy factors. Nature 480: 113-117.

Paul P, van den Hoorn T, Jongsma ML, Bakker MJ, Hengeveld R, Janssen L, Cresswell P, Egan DA, van Ham M, Ten Brinke A, Ovaa H, Beijersbergen RL, Kuijil C, Neefjes J (2011) A genome-wide multidimensional RNAi screen reveals pathways controlling MHC class II antigen presentation. Cell 145: 268-283.

Poulikakos PI, Persaud Y, Janakiraman M, Kong X, Ng C, Moriceau G, Shi H, Atefi M, Titz B, Gabay MT, Salton M, Dahlman KB, Tadi M, Wargo JA, Flaherty KT, Kelley MC, Misteli T, Chapman PB, Sosman JA, Graeber TG, Ribas A, Lo RS, Rosen N, Solit DB (2011) RAF inhibitor resistance is mediated by dimerization of aberrantly spliced BRAF(V600E). Nature 480: $387-390$.

Premsrirut PK, Dow LE, Kim SY, Camiolo M, Malone CD, Miething C, Scuoppo C, Zuber J, Dickins RA, Kogan SC, Shroyer KR, Sordella R, Hannon GJ, Lowe SW (2011) A rapid and scalable system for studying gene function in mice using conditional RNA interference. Cell 145: 145-158.

Rantala JK, Pouwels J, Pellinen T, Veltel S, Laasola P, Mattila E, Potter CS, Duffy T, Sundberg JP, Kallioniemi O, Askari JA, Humphries MJ, Parsons M, Salmi M, Ivaska J (2011) SHARPIN is an endogenous inhibitor of beta1-integrin activation. Nat Cell Biol 13: 1315-1324.

Sax PE (2007) FDA approval: maraviroc. AIDS Clin Care 19: 75. Scholl C, Frohling S, Dunn IF, Schinzel AC, Barbie DA, Kim SY, Silver SJ, Tamayo P, Wadlow RC, Ramaswamy S, Dohner K, Bullinger L, Sandy P, Boehm JS, Root DE, Jacks T, Hahn WC, Gilliland DG (2009) Synthetic lethal interaction between oncogenic KRAS dependency and STK33 suppression in human cancer cells. Cell 137: 821-834.

Sims D, Mendes-Pereira AM, Frankum J, Burgess D, Cerone MA, Lombardelli C, Mitsopoulos C, Hakas J, Murugaesu N, Isacke CM, Fenwick K, Assiotis I, Kozarewa I, Zvelebil M, Ashworth A, Lord CJ (2011) High-throughput RNA interference screening using pooled shRNA libraries and next generation sequencing. Genome Biol 12: R104.

Snuderl M, Fazlollahi L, Le LP, Nitta M, Zhelyazkova BH, Davidson CJ, Akhavanfard S, Cahill DP, Aldape KD, Betensky RA, Louis DN, Iafrate AJ (2011) Mosaic amplification of multiple receptor tyrosine kinase genes in glioblastoma. Cancer Cell 20: 810-817.

Solimini NL, Luo J, Elledge SJ (2007) Non-oncogene addiction and the stress phenotype of cancer cells. Cell 130: 986-988.

Stojdl DF, Lichty B, Knowles S, Marius R, Atkins H, Sonenberg N, Bell JC (2000) Exploiting tumor-specific defects in the interferon pathway with a previously unknown oncolytic virus. Nat Med 6: 821-825.

Stojdl DF, Lichty BD, tenOever BR, Paterson JM, Power AT, Knowles S, Marius R, Reynard J, Poliquin L, Atkins H, Brown EG, Durbin RK, Durbin JE, Hiscott J, Bell JC (2003) VSV strains with defects in their ability to shutdown innate immunity are potent systemic anti-cancer agents. Cancer Cell 4: 263-275.

Zhang Z, Stiegler AL, Boggon TJ, Kobayashi S, Halmos B (2010) EGFRmutated lung cancer: a paradigm of molecular oncology. Oncotarget 1: 497-514.

Zuber J, McJunkin K, Fellmann C, Dow LE, Taylor MJ, Hannon GJ, Lowe SW (2011a) Toolkit for evaluating genes required for proliferation and survival using tetracycline-regulated RNAi. Nat Biotechnol 29: 79-83.

Zuber J, Shi J, Wang E, Rappaport AR, Herrmann H, Sison EA, Magoon D, Qi J, Blatt K, Wunderlich M, Taylor MJ, Johns C, Chicas A, Mulloy JC, Kogan SC, Brown P, Valent P, Bradner JE, Lowe SW, Vakoc CR (2011b) RNAi screen identifies Brd4 as a therapeutic target in acute myeloid leukaemia. Nature 478: 524-528. 